\title{
Caracterização da fauna e flora associada ao sistema de maricultura de macroalgas desenvolvido na praia de Pitangui, Extremo/RN
}

A maricultura da macroalga Gracilaria birdae está sendo desenvolvida de forma experimental na praia de Pitangui, município de Extremoz, RN, com a colocação de balsas flutuantes como estruturas de cultivo a aproximadamente de $200 \mathrm{~m}$ da linha da praia em maré baixa. O presente trabalho objetivou realizar um levantamento preliminar das comunidades da microflora e da fauna acompanhante no cultivo da macroalga Gracilaria birdae, situado na região costeiro marinha de Pitangui, Extremoz/RN. O experimento foi realizado através de coletas sistemáticas no entorno das balsas flutuantes de cultivo da macroalga Gracilaria birdae e zona de arrebentação da praia de Pitangui. As amostras planctônicas foram obtidas com uso de redes de plâncton com malha de 20 e 68 um coletadas à superfície, ao redor de uma balsa de cultivo recém-instalada com $9 \mathrm{~m}$ de extensão. O material planctônico coletado foi fixado em solução de formol a $4 \%$ e identificado em microscópio óptico, com auxílio de chaves de identificação do plâncton marinho. A ictiofauna foi coletada com o uso de uma rede de arrasto do tipo 'picaré', com $4 \%$ e identificado em microscópio óptico, com auxílio de chaves de identificação do plâncton marinho. A ictiofauna foi coletada com o uso de uma rede de arrasto do tipo 'picare', com
dimensões de $20 \mathrm{~m}$ de comprimento, $1,5 \mathrm{~m}$ de altura e $5 \mathrm{~mm}$ de malha entre nós. A comunidade fitoplânctonica foi composta pelos gêneros Bellerochea sp., Odontella sp., Striatella sp., Coscinodiscus sp., Fragilaria sp., Hemidiscus, sp. Rhabdonema sp., Triceratium sp., Campylodiscus sp., Chaetoceros sp e Asterionella sp. Já a comunidade zooplanctônica apresentou: Foraminifera, Copepoda: Oithona sp e Corycaeus sp.; Euterpina acutifrons; Parvocalanus crassirotris, Paracalanus quasimodo e Acartia lilljeborgi; Larva Cifonauta (Bryozoa) e Larva de Bivalve (Mollusca). A ictiofauna capturada compreendeu um total de 333 indivíduos, dentre os quais se identificou representantes de sete ordens, 15 famílias e 25 espécies. As ordens identificadas foram Perciformes $(56 \%)$, Clupeiformes (12\%), Pleuronectiformes (12\%), Siluriformes (8\%), Albuliformes (4\%), Beloniformes $(4 \%)$ e Tetraodontiformes $(4 \%)$. Essas análises preliminares auxiliarão em estudos posteriores relacionados à análise de impactos à diversidade e riqueza do plâncton decorrentes do cultivo da macroalga Gracilaria birdae, em sistema de cultivo do tipo balsa, além de registros da ictiofauna para zona de arrebentação da praia de Pitangui.

\section{Characterization of fauna and flora associated with the seaw eed mariculture system developed on Pitangui, Extremoz/RN beach}

\begin{abstract}
Gracilaria birdae seaweed mariculture is being developed experimentally on Pitangui beach, Extremoz, RN, with floating rafts as cultivation structures approximately $200 \mathrm{~m}$ from the beach at low tide. The present work aimed to carry out a preliminary survey of the microflora communities and the accompanying fauna in the Gracilaria birdae macroalgae cultivation, located in the coastal coastal region of Pitangui, Extremoz / RN. The experiment was carried out through systematic collections around the floating rafts of Gracilaria birdae macroalgae and Pitangui beach surf zone. Planktonic samples were obtained using 20 and $68 \mu \mathrm{m}$ mesh plankton nets collected at the surface around a newly installed $9 \mathrm{~m}$ long raft. The collected planktonic material was fixed in $4 \%$ formaldehyde solution and identified under optical microscope, with the help of marine plankton identification keys. The ichthyofauna was collected using a 'picaré' trawl, with dimensions of $20 \mathrm{~m}$ in length, $1.5 \mathrm{~m}$ in height and $5 \mathrm{~mm}$ mesh between nodes. The phytoplankton community was composed of the genera Bellerochea sp., Odontella sp., Striatella sp., Coscinodiscus sp., Fragilaria sp., Hemidiscus, sp. Rhabdonema sp., Triceratium sp., Campylodiscus sp., Chaetoceros sp. And Asterionella sp. The zooplankton community presented: Foraminifera, Copepoda: Oithona sp and Corycaeus sp .; Euterpine acutifrons; Parvocalanus crassirotris, Paracalanus quasimodo and Acartia lilljeborgi; Cifonaut Larva (Bryozoa) and Bivalve

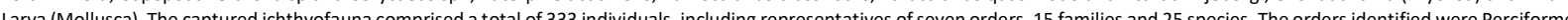
Larva ( $(56 \%)$, Clupeiformes (12\%), Pur in studies related to the analysis of impacts on plankton diversity and richness resulting from Gracilaria birdae macroalgae cultivation in a balsa-type cultivation system, as well as ichthyofauna records for the Pitangui beach surf zone.
\end{abstract}

Keywords: Surf Zone; Ichthyology; Mariculture.

Topic: Desenvolvimento, Sustentabilidade e Meio Ambiente

Reviewed anonymously in the process of blind peer
Received: 10/08/2019

Approved: 28/09/2019
Laiz Araújo Silva do Nascimento (iD)

Universidade Federal do Rio Grande do Norte, Brasil

http://lattes.cnpq.br/6941289315907222

http://orcid.org/0000-0001-6492-0859

laiz nascimento@outlook.com

Dárlio Inácio Alves Teixeira (iD)

Universidade Federal do Rio Grande do Norte, Brasil

http://lattes.cnpq.br/6835210577941969

http://orcid.org/0000-0002-6504-1802

darlioteixeira@gmail.com

Fábio Magno da Silva Santana

Universidade Federal do Rio Grande do Norte, Brasil

http://lattes.cnpq.br/5346671879489841

fabsantana@yahoo.fr

d

DOI: 10.6008/CBPC2179-6858.2019.005.0022
Cibele Soares Pontes (iD)

Universidade Federal do Rio Grande do Norte, Brasil

http://lattes.cnpq.br/3943018673158703

http://orcid.org/0000-0003-1993-0032

cibelepontes.ufrn@yahoo.com.br

\section{Referencing this:}

NASCIMENTO, L.; TEIXEIRA, D. I.; SANTANA, F.; PONTES, C.

Caracterização da fauna e flora associada ao sistema de maricultura de macroalgas desenvolvido na praia de Pitangui, Extremo/RN. Revista Ibero Americana de Ciências Ambientais, v.10, n.5, p.250258, 2019. DOI: http://doi.org/10.6008/CBPC2179-

$\underline{6858.2019 .005 .0022}$ 


\section{INTRODUÇÃO}

Este trabalho foi desenvolvido e executado como parte do projeto de extensão Maricultura de Macroalgas no Nordeste do Brasil: Ações em continuidade, que foca suas ações na praia e comunidade de Pitangui. O programa visa o fortalecimento e desenvolvimento das atividades de maricultura de macroalgas marinhas e seus valores agregados, apoiando dezenas de famílias e indiretamente centenas de pessoas, favorecendo melhores condições socioambientais ao público envolvido e ao ecossistema costeiro-marinho, desde 2010.

A Associação de Maricultura e Beneficiamento de Algas de Pitangui - AMBAP, localizada em Extremoz/RN, já tem acumulado alguns conhecimentos sobre o processo produtivo de macroalgas, produtoras da AMBAP desenvolvem o cultivo de G. birdiae usando a metodologia de balsas flutuantes, este grupo já possui informações e de práticas sobre alimentos e cosméticos a base de algas. A partir das ações em desenvolvimento pelo Programa PROEXT 2014, com continuidade em 2015 e 2016, este grupo produtivo avançou no processo de aplicação da tecnologia social do cultivo de macroalgas marinhas através de apoio do PROEXT durante os anos 2015 e 2016, ocorreram alguns avanços neste processo, Silva (2014) demonstrou a viabilidade técnica do uso da balsa flutuante como tecnologia social no desenvolvimento de um cultivo experimental em Pitangui-Extremoz/RN.

A maricultura da macroalga Gracilaria birdae está sendo desenvolvida de forma experimental na praia de Pitangui, município de Extremoz, estado do Rio Grande do Norte, distante $25 \mathrm{~km}$ de Natal-RN, entre as coordenadas $05^{\circ} 37^{\prime} 19.4^{\prime \prime}$ S e $35^{\circ} 13^{\prime} 29.3^{\prime \prime}$ W, uma distância aproximadamente de $100 \mathrm{~m}$ da linha da praia, com a colocação de balsas flutuantes como estruturas de cultivo. Este é realizado através de propagação vegetativa em estruturas de balsas flutuantes que é constituída por uma corda principal onde são penduradas cordas secundárias. Para a sua instalação em mar, as extremidades são fixadas através de blocos de concreto (poitas) para evitar ou minimizar danos durante o cultivo (SILVA, 2014).

A zona de arrebentação é um ambiente importante por constituir uma área de manutenção da vida marinha, com a ictiofauna presente representando três quartos das espécies conhecidas. Além disso, o uso desses recursos e a influência de cidades situadas próximo à costa podem ser as causas dos impactos sobre essas espécies (FIGUEIREDO et al., 2015).

Como a estrutura do cultivo da macroalga G. birdiae fica muito próxima à zona de arrebentação da praia de Pitangui e essas zonas são importantes áreas para o recrutamento de peixes, baseado em Santana et al. (2013). Estudos sobre a ictiofauna de praia mostram a presença de várias espécies, principalmente no estágio juvenil, o que indica a importância deste ambiente como berçário (ROBERTSON et al., 1984; GODEFROID et al., 2001; SANTANA et al., 2013). Considerando que a zona de arrebentação é uma importante região de transição para o recrutamento de larvas eclodidas em áreas próximas à costa, por se tratar de ambiente de alta energia.

O litoral do Estado do Rio Grande do Norte é um dos mais famosos e conhecidos do Brasil, com uma extensão aproximada de quatrocentos e vinte quilômetros. É caracterizado por praias arenosas que se 
estendem desde a cidade de Tibau do Sul a Baía Formosa e são intensamente utilizadas pela população humana como fonte de renda através da pesca artesanal, para lazer e recreação (CAVALCANTI et al., 2004).

Atualmente ainda são escassas informações sobre a riqueza e a distribuição das espécies de peixes que ocorrem na extensa costa do litoral brasileiro. Existem poucas fontes sistematizadas que disponibilizem tais dados, sob a forma de registro da ocorrência, preferência espacial, características diagnósticas e biológicas (GARCIA JÚNIOR et al., 2010).

Em zonas de arrebentação de praias de alta energia, encontra-se elevada concentração de produtores primários (fitoplâncton e fitobentos), os quais constituem suprimento alimentar básico para diversos consumidores primários dos ecossistemas costeiros e marinhos adjacentes. Nestas zonas, os processos de ressuspensão provocados pelos ventos, arrebentação das ondas e correntes litorâneas são responsáveis pelo intercâmbio de recursos na interface sedimento/água na qual as microalgas bentônicas passam a fazer parte do plâncton, alterando, desta forma, a estrutura da comunidade fitoplanctônica local (SOUSA et al., 2009).

O zooplâncton pode ser dividido em duas categorias com base no seu tempo de permanência na comunidade planctônica, sendo elas o holoplâncton, também chamado de plâncton permanente, sendo o organismo que permanece plâncton durante toda a sua vida, e o meroplâncton, conhecido também como zooplâncton temporário, que permanecem apenas temporariamente na comunidade planctônica, como, por exemplo, as larvas de algumas espécies crustáceas e peixes (PEREIRA et al., 2002).

Assim o estudo do plâncton é de importância prioritária, pois, enquanto o fitoplâncton produz a matéria orgânica pela fotossíntese, o zooplâncton constitui um elo importante na transferência de energia na forma fitoplâncton-bacterioplâncton ou, na de detritos orgânicos particulados para os demais níveis tróficos, incluindo moluscos, crustáceos e peixes de interesses comerciais. Influenciam e determinam espécies nectônicas e bentônicas que têm estágios no plâncton, além de atuar na ciclagem de energia de um ambiente para outro (GROSS et al., 1996).

Os estudos sobre a fauna da região nordestina não têm sido intensivos, observando-se, no entanto, esforços isolados para o seu conhecimento (CAVALCANTI et al., 2004). Sendo o atual trabalho de levantamento de espécies de fauna e flora de fundamental relevância para o enriquecimento dos estudos para a região nordestina. Uma vez que, em seu conjunto, o plâncton é de vital importância para os ecossistemas marinhos, pois representa a base da teia alimentar pelágica nos oceanos e mudanças em sua composição e estrutura podem ocasionar profundas modificações em todos os níveis tróficos (YONEDA, 1999).

Assim, os dados gerados a partir dessa proposta de trabalho são ser importantes para subsidiar a pesca artesanal da localidade, visto que identificamos as espécies desse setor produtivo, mostrando com dados técnico-científicos a diversidade de espécies do ecossistema marinho local. Dessa forma, objetivou-se com esse trabalho caracterizar as espécies de fito e zooplâncton que ocorrem na zona associada as balsas de cultivo da Gracilaria birdiae atualmente instaladas na praia de Pitangui-Extremoz/RN, assim como identificar as espécies de ictiofauna que ocorrem na zona de arrebentação da mesma. 


\section{METODOLOGIA}

O estudo foi realizado na praia de Pitangui, situada no município de Extremoz, porção leste do estado do Rio Grande do Norte, distante $25 \mathrm{~km}$ de Natal (Figura 1). Esta área pertence à Bacia Potiguar, tal plataforma continental é relativamente ampla (aproximadamente 20 a $30 \mathrm{~km}$ de largura) e de baixa profundidade (em geral inferior a $30 \mathrm{~m}$ ), alcançando o talude entre 40 e $100 \mathrm{~m}$. O cultivo da macroalga Gracilaria birdae fica localizado a 100 metros da praia de Pitangui.

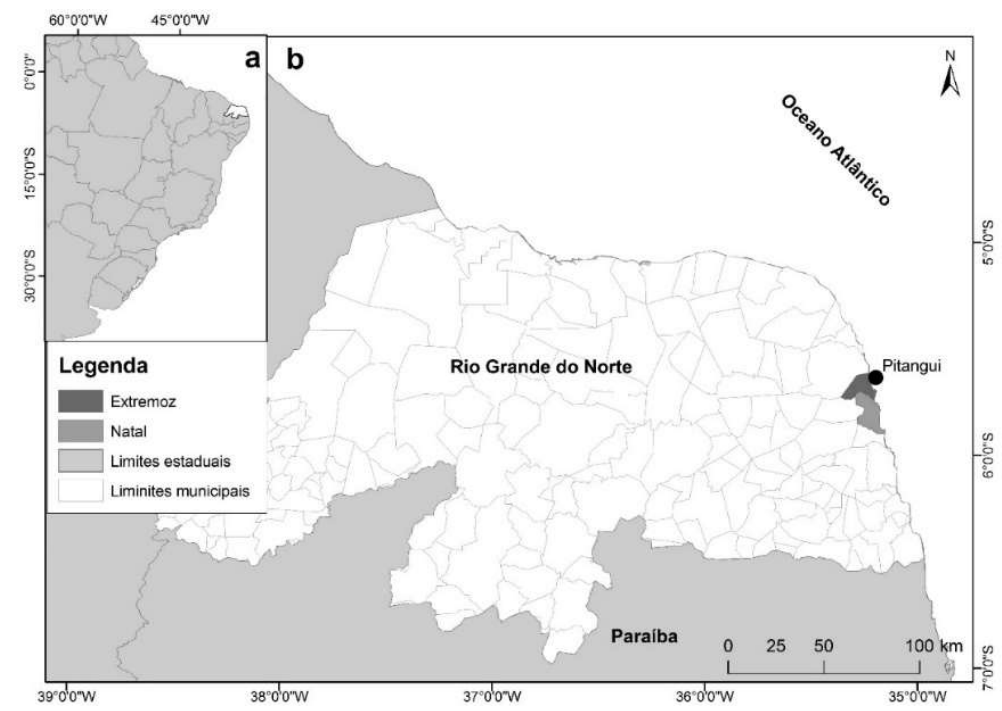

Figura 1: Mapa da região amostrada, destacando Natal, a capital do estado e o local de coleta na praia de Pitangui no município de Extremoz/RN.

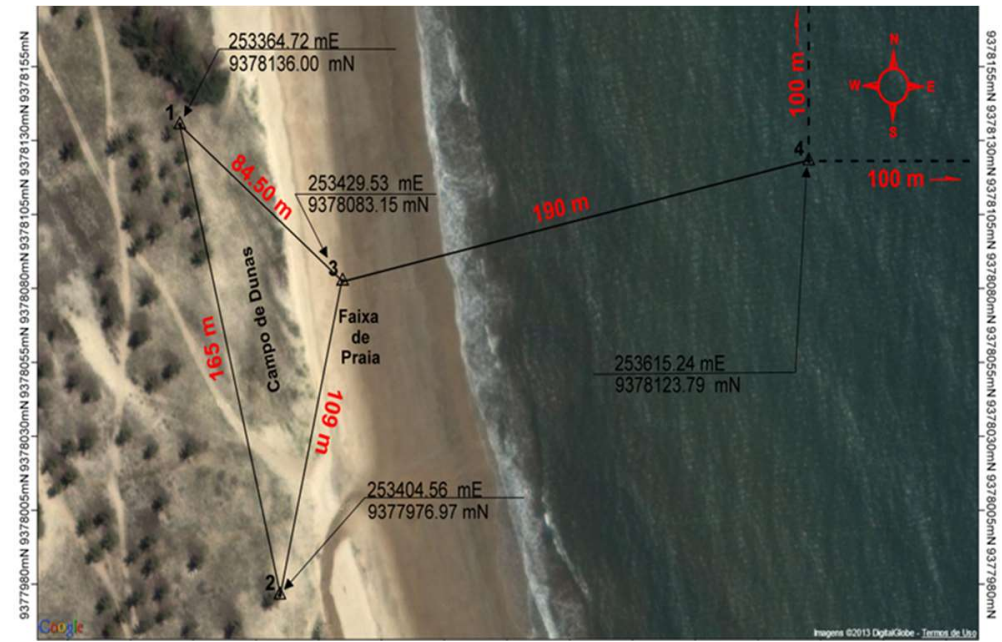

Figura 2: Localização do cultivo da G. birdiae na praia de Pitangui-RN. Fonte: Silva (2014).

Para o estudo do fito e zooplâncton associado ao cultivo, as amostras foram obtidas com o uso de redes de plâncton com malha de 20 e $68 \mu \mathrm{m}$ coletadas a superfície, no entorno de uma balsa flutuante de cultivo da macroalga Gracilaria recém-instalada na data de 24 de novembro de 2018. 0 material foi fixado em solução de formol a $4 \%$ na proporção de 1:1. A mostra foi encaminhada para o laboratório de limnologia da Escola Agrícola de Jundiaí - UFRN, onde, com o auxílio de pipeta e utilização de lâmina e lamínula a amostra foi analisada em microscópio óptico.

As espécies de ictiofauna foram capturadas na zona de arrebentação da praia de Pitangui-ExtremozRN (05 $\left.37^{\prime} 50.1^{\prime \prime} \mathrm{S} 35^{\circ} 13^{\prime} 15.7^{\prime \prime} \mathrm{W}\right)$ durante a baixa-mar, com a utilização de rede de arrasto do tipo 'picaré' de 
20 metros de comprimento e 1,5 metro de altura, nos meses de novembro de 2018 e agosto de 2019. Os arrastos foram realizados três vezes seguidas num mesmo ponto da zona de arrebentação. Os espécimes capturados foram armazenados em sacos plásticos separados por arrasto (arrasto 1, 2 ou 3), em seguida armazenados em bombonas com gelo e transportados para o Laboratório de Processamento de Pescado da Escola Agrícola de Jundiaí-UFRN, situada em Macaíba.

Nos Laboratórios de Aquicultura da Escola Agrícola de Jundiaí (EAJ)/Unidade Acadêmica Especializada em Ciências Agrárias, da Universidade Federal de Rio Grande do Norte (UFRN), foi realizada a identificação taxonômica dos exemplares capturados e a tomada de dados biométricos. Foi medido o comprimento padrão (CP) e comprimento total (CT) dos indivíduos em centímetros. No laboratório os peixes foram pesados e medidos. Foram analisadas as características morfológicas e realizadas medições morfométricas e contagens merísticas dos peixes objetivando a identificação taxonômica das espécies em estudo. Principais medidas morfométricas e merísticas dos peixes ósseos utilizadas na identificação das espécies são: CT - comprimento total e CP - comprimento padrão. A identificação da fauna se deu até o nível de espécie, baseando-se em Araújo et al. (2004) e Carpenter (2002). Para a análise dos dados, os espécimes de ictiofauna capturados foram identificados, listados e contabilizados de acordo com o arrasto no qual foram capturados.

\section{RESULTADOS}

A comunidade fitoplanctônica foi composta pelos seguintes gêneros Bellerochea sp., Odontella sp., Striatella sp., Coscinodiscus sp., Fragilaria sp., Hemidiscus sp., Rhabdonema sp., Triceratium sp., Campylodiscus sp., Chaetoceros sp e Asterionella sp (Tabela 1). As diatomáceas (filo Bacillariophyta), foram os organismos mais representantes da amostra oriunda do cultivo de macroalgas de Pitangui (Bellerochea sp., Odontella sp., Striatella sp., Coscinodiscus sp., Fragilaria sp., Hemidiscus sp., Rhabdonema sp., Triceratium $S P$, Chaetoceros sp e Asterionella sp.).

Tabela 1: Lista das espécies/gêneros de Fitoplâncton e Zooplâncton que foram identificados na Praia de Pitangui/RN.

\begin{tabular}{|l|l|}
\hline Fitoplâncton & Zooplâncton \\
\hline Bellerochea $s p$. & Foraminifera \\
\hline Odontella $s p$. & Oithona $s p$. \\
\hline Striatella $s p$. & Corycaeus sp. \\
\hline Coscinodiscus $s p$. & Euterpina acutifrons \\
\hline Fragilaria $s p$. & Parvocalanus crassirotris \\
\hline Hemidiscus $s p$. & Paracalanus quasimodo \\
\hline Rhabdonema $s p$. & Acartia lilljeborgi \\
\hline Triceratium $s p$. & Larva Cifonauta \\
\hline Campylodiscus $s p$. & Larva de Bivalve \\
\hline Chaetoceros $s p$. & \\
\hline Asterionella $s p$. & \\
\hline
\end{tabular}

A comunidade zooplanctônica apresentou: Copepoda: Oithona sp. e Corycaeus sp.; Euterpina acutifrons; Parvocalanus crassirotris; Paracalanus quasimodo e Acartia lilljeborgi e Larva Cifonauta (Bryozoa) (Tabela 1). O grupo predominante na análise qualitativa desse trabalho foi Copepoda, pertencente ao holoplâncton marinho, apresentando sete diferentes gêneros/espécies. Enquanto o meroplâncton foi pouco 
representativo, apresentando apenas larva de Bryzoa.

A ictiofauna capturada compreendeu um total de 333 indivíduos, dentre os quais se identificou representantes de sete ordens, 15 famílias e 25 espécies. As ordens identificadas foram Perciformes (56\%), Clupeiformes (12\%), Pleuronectiformes (12\%), Siluriformes (8\%), Albuliformes (4\%), Beloniformes (4\%) e Tetraodontiformes (4\%).

A ordem Perciformes esteve representada por sete famílias e 14 espécies. Dentro desta ordem, a família Sciaenidae (Figura 3), representada por Ophioscion punctatissimus, Larimus breviceps Cuvier, 1830, Stellifer rastrifer (Jordan, 1889), Isopisthus parvipinnis Cuvier, 1830, e Menticirrhus littoralis Holbrook, 1847 foi a que se destacou, representando 63,66\% de todas as amostras com 212 indivíduos (Tabela 2).

Tabela 2: Lista de espécies identificadas coletadas da praia de Pitangui, Extremoz-RN. Ordem, Família, Espécie, quantidade (n) e Nome Popular.

\begin{tabular}{|l|l|l|l|l|}
\hline Ordem & Familia & Espécie & $\mathbf{n}$ & Nome Popular \\
\hline Albuliformes & Albulidae & Albula vulpes & 2 & Ubarana-focinho-de-rato \\
\hline Clupeiformes & Pristigasteridae & Chirocentrodon bleekerianus & 1 & Arenque \\
\hline Clupeiformes & Pristigasteridae & Pellona harroweri & 3 & Sardinha \\
\hline Clupeiformes & Engraulidae & Anchoviella lepidentostole & 29 & Ginga \\
\hline Siluriformes & Ariidae & Hexanematichthys grandoculis & 2 & Bagre \\
\hline Siluriformes & Ariidae & Genidens genidens & 1 & Bagre \\
\hline Beloniformes & Hemiramphidae & Hyporhamphus unifasciatus & 1 & Peixe-agulha \\
\hline Perciformes & Scombridae & Scomberomorus brasiliensis & 1 & Peixe-serra \\
\hline Perciformes & Carangidae & Selene vômer & 2 & Peixe-galo \\
\hline Perciformes & Carangidae & Caranx latus & 1 & Xaréu \\
\hline Perciformes & Carangidae & Trachinotus carolinus & 2 & Pampo \\
\hline Perciformes & Gerreidae & Ulaema lefroyi & 1 & Carapicu \\
\hline Perciformes & Heamulidae & Conodon Nobilis & 23 & Roncador \\
\hline Perciformes & Heamulidae & Haemulopsis corvinaeformis & 21 & Coró \\
\hline Perciformes & Polynemidae & Polydactylus virginicus & 23 & Barbudo \\
\hline Perciformes & Sciaenidae & Ophioscion punctatissimus & 114 & Mulato \\
\hline Perciformes & Sciaenidae & Larimus breviceps & 40 & Boca-mole \\
\hline Perciformes & Sciaenidae & Stellifer rastrifer & 56 & Cangoá \\
\hline Perciformes & Sciaenidae & Isopisthus parvipinnis & 1 & Pescada \\
\hline Perciformes & Sciaenidae & Menticirrhus littoralis & 1 & Papa-terra \\
\hline Perciformes & Trichiuridae & Trichiurus lepturus & 4 & Espada \\
\hline Pleuronectiformes & Paralichthyidae & Etropus crossotus & 1 & Sóia \\
\hline Pleuronectiformes & Cynoglossidae & Symphurus plagusia & 1 & Sóia \\
\hline Pleuronectiformes & Cynoglossidae & Symphurus tesselatus & 1 & Sóia \\
\hline Tetraodontiformes & Tetraodontidae & Sphoeroides testudineus & 1 & Baiacu \\
\hline
\end{tabular}

Outra família com alta representatividade, dentre os Perciformes e no total, foi Heamulidae, com 44 indivíduos. Esta família apresentou duas espécies, Conodon nobilis e Haemulopsis corvinaeformis e foi responsável por $13,2 \%$ do total das amostras (Tabela 2). Outra família com alta representatividade, dentre os Perciformes foi Polynemidae, com 23 indivíduos. Esta família apresentou apenas uma espécie, Polydactylus virginicus e foi responsável por 6,9\% do total das amostras (Tabela 2).

A família Engraulidae (Clupeiformes) foi composta pela espécie Anchoviella lepidentostole, sendo a quarta família em número de indivíduos, 29, representando $8,7 \%$ do total de indivíduos coletados. A família Carangidae apresentou 5 (1,5\%) indivíduos no total e esteve representada por Trachinotus carolinus, Caranx latus e Selene vomer. A porcentagem das famílias Ariidae, Hemiramphidae, Scombridae, Gerreidae, Paralichthyidae, Cynoglossidae e Tetraodontidae foi de 3\%, visto que estas somente estiveram representadas por apenas um ou dois exemplares cada. A espécie Ophioscion punctatissimus, da família Sciaenidae, foi a 
mais representativa, com 114 indivíduos. Esta contabilizou 34,23\% do total de peixes coletados.

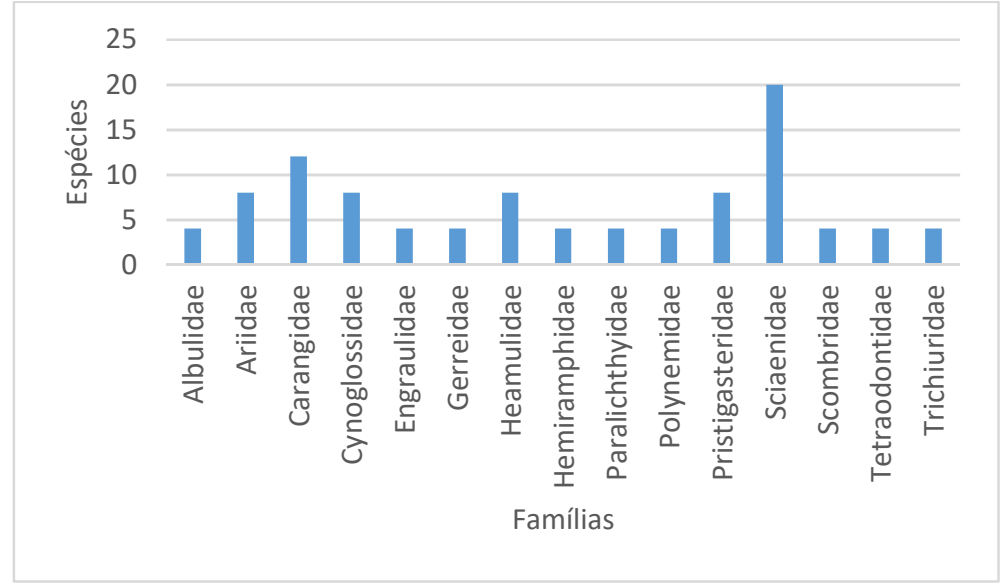

Figura 3: Distribuição das espécies por família.

\section{DISCUSSÃO}

A partir dos dados obtidos com esse trabalho, foi possível observar nas amostras de plâncton oriundas da praia de Pitangui-Extremoz/RN seis espécies zooplanctônicas, sendo predominante o holoplâncton, e 6 espécies de fitoplâncton. 0 grupo predominante na análise do zooplâncton foi Copepoda, pertencente ao holoplâncton marinho, apresentando sete diferentes gêneros/espécies. Enquanto o meroplâncton foi pouco representativo, apresentando apenas larva de Bryzoa.

Dessa forma, os organismos holoplanctônicos se caracterizaram por maior representatividade sobre os demais grupos da comunidade macrozooplanctônica coletada. Sendo as diatomáceas mais representativas na análise fitoplanctõnica nesse estudo, é importante ressaltar que elas têm sido descritas como representativas de águas costeiras frequentemente associadas a regiões de frentes oceânicas com elevado estresse causado por uma coluna d'água não estratificada, turbulenta e sob a influência de correntes costeiras com altas velocidades (SOUSA et al., 2009).

As diatomáceas constituem um dos principais grupos do fitoplâncton em águas marinhas neríticas e estuarinas (BRANDINI et al., 1996). Geralmente compreendem grande parte da biomassa do fitoplâncton, evidenciando seu papel na teia trófica pelágica e nos fluxos de carbono na região de estudo (BRANDINI et al., 2001).

Além disso, a comunidade planctônica apresenta um caráter muito dinâmico, com elevadas taxas de reprodução e perda, respondendo rapidamente às alterações físicas e químicas do meio aquático e estabelecendo complexas relações intra e interespecíficas na competição e utilização do espaço e dos recursos (VALIELA, 1995).

O primeiro catálogo dos peixes do litoral, do Estado do Rio Grande do Norte, Brasil (Osteichthyes e Chondrichthyes), foi publicado em 1988, onde foram registradas 20 ordens, 73 famílias e 190 espécies de peixes marinhos (SOARES, 1988). A maior representatividade da ordem Perciformes corrobora as afirmações de Vasconcelos Filho et al. (2004). Dentre as famílias desta ordem, a mais representativa foi a Sciaenidae, seguida de Polynemidae e Carangidae. 
A ordem Perciformes foi à ordem mais representativa corroborando com os registros de ocorrência e predominância desta ordem na costa do Rio Grande do Norte (GARCIA JR., 2006). De acordo com Menezes et al. (1980), Ophioscion punctatissimus vive em fundos lodosos e arenosos, em águas costeiras de pouca profundidade. Menezes et al. (1980) afirmam que a espécie Ophioscion punctatissimus é pouco comum no litoral brasileiro, contudo, o presente trabalho mostra que essa espécie teve significativa abundância em relação as outras nas coletas realizadas.

\section{CONCLUSÕES}

Trabalhos como este de listagens de espécies são importantes para conhecer a biodiversidade local, principalmente quando se trata de espécies nocivas e/ou exóticas que possam causar danos ao ambiente que impactam. Essas análises auxiliarão em estudos posteriores relacionados à análise de impactos à diversidade e riqueza do plâncton decorrentes do cultivo da macroalga Gracilaria birdae, em sistema de cultivo do tipo balsa, além de registros da ictiofauna para zona de arrebentação da praia de Pitangui. Os resultados deste trabalho poderão agregar material e contribuir no estudo das características usadas para reconhecer cada uma das espécies de peixes marinhos da região, através dos desenhos representativos de cada peixe.

\section{REFERÊNCIAS}

ARAÚJO, M. E.; TEIXEIRA, J. M. C.; OlIVEIRA, A. M. E.. Peixes Estuarinos Marinhos do Nordeste Brasileiro: Guia Ilustrado. Fortaleza: UFC, 2004.

BRANDINI, F. P.; FERNANDES, L. F.. Microalgae ofthe continental shelf off Paraná state, southeastern Brazil: a review of studies. Oceanogr, 1996.

BRANDINI, F. P.; SILVA, E. T.; PELIZZARI, F. M.; FONSECA, A. L. O.; FERNANDES, L. F.. Production and biom-ass accumulation of periphytic diatoms growing on glassslides during a 1-year cycle in a subtropical estuarineenvironment (Bay of Paranaguá, southern Brazil). Mar. Biol., v.138, p.163-171, 2001.

CAVALCANTI, E. A. H.; LARRAZÁBAL, M. E. L..

Macrozooplâncton da Zona Econômica Exclusiva do Nordeste do Brasil (segunda expedição oceanográfica REVIZEE/NE II) com ênfase em Copepoda (Crustacea). Revista Brasileira de Zoologia, v.21, n.3, p.467-475, 2004. DOI: http://dx.doi.org/10.1590/S0101-81752004000300008

CARPENTER, K. E.. The living marine resources of the Western Central Atlantic: Bony fishes part 1 (Ophistognathidae to Molidae), sea turtles and marine mammals. Rome: FAO, 2002.

FIGUEIREDO, G. G. A. A.; PESSANHA, A. L. M.. Comparative study of trophic organization of juvenile fish assemblages of three tidal creeks in a tropical semi-arid estuary. J. Fish.

Biol., v.89, n.1, p.680-95, 2015. DOI:

http://doi.org/10.1111/jfb.12844

GARCIA JR, J.. Inventário das espécies de peixes da costa do Estado do Rio Grande do Norte e aspectos zoogeográficos da ictiofauna recifal do Oceano Atlântico. Dissertação (Mestrado) - Universidade Federal do Rio Grande do Norte, Natal, 2006.

GODEFROID, R. S.; SANTOS, C.; HOFSTAETTER, M.; SPACH, H. L.. Occurrence of Larvae and Juveniles of Eucinostomusargenteus, Eucinostomusgula, Menticirrhus americanus, Menticirrhus littoralis, Umbrina coroides and Micropogoniasfurnieri at Pontal do Sul beach, Paraná. Brazilian. Archives of Biology and Technology, v.44, n.4, p.411-18, 2001.

GROSS, M. G.; ROSS, E. G.. Oceanography, a view of earth. New Jersey: Prentice Hall, 1996.

GARCIA JÚNIOR, G. J.; MENDES, L. F.; SAMPAIO, C. L. F.; LINS, J. E.. Biodiversidade Marinha da Bacia Potiguar: Ictiofauna. Rio de Janeiro: Museu Nacional, 2010.

PEREIRA, R. C.; GOMES, S.. A Biologia Marinha. Rio de Janeiro: Interciência, 2002.

ROBERTSON, A. I.; LENANTON, R. C. J.. Fish community stucture and food chain dynamics in the surf-zone of sandy beaches: the role of detached macrophyte detritus. Journal of Experimental Marine Biology and Ecology, v.84, p.26583, 1984.

SANTANA, F. M. S.; SEVERI, W.; FEITOSA, C. V.; ARAÚJO, M. $E$.. The influence of seasonality on fish life stages and residence in surf zones: a case of study in a tropical region. Biota Neotrop., v.13, n.3, 2013. DOI: http://dx.doi.org/10.1590/S1676-06032013000300021

SILVA, L. V.. Avaliação da Taxa de Crescimento da 
Macroalga Gracilaria birdiae na Zona Costeira de PitanguiExtremoz/RN e acompanhamento do teor de proteína e carboidrato desta espécie ao longo do cultivo. Monografia (Graduação em Engenharia de Aquicultura) - Universidade Federal do Rio Grande do Norte, Natal, 2014.

SOARES, L. H.. Catálogo dos peixes do litoral do Estado do Rio Grande do Norte, Brasil (Osteichthyes e Chondrichthyes) Boletim do Departamento de Oceanografia e Limnologia do Centro de Biociências da Universidade Federal do Rio Grande do Norte, Natal, v.7, p.1-39, 1988.

SOUSA, E. B.; COSTA, V. B.; PEREIRA, L. C. C.; COSTA R. M. Variação temporal do fitoplâncton e dos parâmetros hidrológicos da zona de arrebentação da Ilha Canela
(Bragança, Pará, Brasil). Acta Botanica Brasilica, v.23, n.4, 2009. DOI: http://dx.doi.org/10.1590/S010233062009000400018

VALIELA, I.. Marine ecological processe. 2 ed. New York Springer-Verlag, 1995.

VASCONCELOS FILHO, A. L.; GUEDES, D. S.; TEIXEIRA, S. F.; OLIVEIRA, A. M. E.. Peixes marinhos costeiros e estuarinos. In: LEITÃO, S. N.; ESKINAZI-LEÇA, E.; COSTA, M.

Oceanografia: um cenário tropical. Recife: Bagaço, 2004. p.555-570.

YONEDA, N. T.. Área Temática: Plâncton. Curitiba: Centro de Estudos do Mar, 1999.

A CBPC - Companhia Brasileira de Produção Científica (CNPJ: 11.221.422/0001-03) detém os direitos materiais desta publicação. Os direitos referem-se à publicação do trabalho em qualquer parte do mundo, incluindo os direitos às renovações, expansões e disseminações da contribuição, bem como outros direitos subsidiários. Todos os trabalhos publicados eletronicamente poderão posteriormente ser publicados em coletâneas impressas sob coordenação da Sustenere Publishing, da Companhia Brasileira de Produção Científica e seus parceiros autorizados. Os (as) autores (as) preservam os direitos autorais, mas não têm permissão para a publicação da contribuição em outro meio, impresso ou digital, em português ou em tradução. 